\title{
Análisis de la relación entre la innovación y la gestión del conocimiento con la competitividad empresarial en una muestra de empresas en la ciudad de Bogotá
}

\author{
CÉSAR AUGUSTO BERNAL TORRES, D.B.A..,2 \\ Profesor Asociado, Universidad de La Sabana, Colombia. \\ cesar.bernal@unisabana.edu.co \\ GERMÁN FRACICA NARANJO, MBA \\ Profesor de postgrados, Universidad de La Sabana, Colombia. \\ german.fracica@gmail.com
}

JOSÉ SALOMÓN FROST GONZÁLEZ, MBA

Director del Instituto de Postgrados-Fórum, Universidad de la Sabana, Colombia.

Salomon.frost@unisabana.edu.co

\section{RESUMEN}

Este estudio analiza la relación directa entre las actividades de innovación y las de gestión del conocimiento con la capacidad competitiva organizacional. Los resultados indican que, para el caso de una muestra de 53 empresas estudiadas en la ciudad de Bogotá, no hay evidencia de esa relación. Estos resultados son motivo para la reflexión académica, empresarial y del gobierno sobre el tema, si se tiene en cuenta que son diversos y reiterados los escenarios en los que se hace énfasis sobre la necesidad de implementar actividades de innovación y de gestión del conocimiento para potenciar la capacidad competitividad empresarial y nacional, dando por supuesta la existencia de relación directa entre estas variables, lo cual no siempre es así.

Palabras clave. Gestión del conocimiento; innovación; competitividad empresarial.

Recibido: 29-jun-11, corregido: 27-abr-12 y aceptado: 26-jun-12

Clasificación JEL: Moo

@

\footnotetext{
1 Este documento fue seleccionado en la convocatoria para enviar artículos, Call for Papers, realizada en el marco del “II Simposio Iberoamericano de Estudios Gerenciales: Una mirada interdisciplinar a la innovación”, organizado por la revista académica Estudios Gerenciales bajo la dirección de la Facultad de Ciencias Administrativas y Económicas de la Universidad Icesi; el evento tuvo lugar los días 12, 13 y 14 de octubre de 2011, en la ciudad de Cali (Colombia). Este documento fue presentado en las sesiones simultáneas del área de "Modelos de innovación aplicados".

2 Autor para correspondencia. Dirigir a: Universidad de la Sabana, Oficina 217, Edificio E2 del Campus del

Puente del Común Km 7, Autopista Norte de Bogotá, Chía, Cundinamarca.
} 
Análisis de la relación entre la innovación y la gestión del conocimiento con la competitividad empresarial en una muestra de empresas en la ciudad de Bogotá

\section{Analysis of the relationship between innovation and knowledge management with organizational competitiveness in a sample of companies in Bogota}

This study analyzes the relationship between innovation and knowledge management activities and organizational competitive capacity. The results indicate that in the case of a sample of 53 companies studied in the city of Bogota, there was no evidence of such a relationship. These results also encourage a reflection on this topic on the part of academics, business owners, and government officials, taking into account that there are diverse repeated scenarios where emphasis is made on the need for implementing innovation and knowledge management initiatives that boost organizational and national competitiveness capacity, based on the assumption that there is a direct relationship between these variables - but this does not always hold true.

Keywords. Knowledge management; innovation; corporate competitiveness.

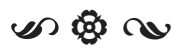

\section{Análise da relação entre inovação e gestão do conhecimento para a competitividade empresarial em uma amostra de empresas em Bogotá}

Este estudo analisa a relação entre as atividades de inovação e a gestão do conhecimento com competitividade organizacional. Os resultados indicam que, para o caso de uma amostra de 53 empresas estudadas na cidade de Bogotá, não há nenhuma evidência desse relacionamento. Estes resultados são levam a reflexão acadêmica, empresarial e do governo cerca da questão, tendo em conta que são cenários diferentes e repetidos em que a ênfase é sobre a necessidade de implementar as atividades de inovação e gestão do conhecimento para melhorar a capacidade e competitividade nacional, assumindo a existência de uma relação direta entre essas variáveis, o que não é sempre assim.

Palavras-chave. Gestão do conhecimento; inovação; competitividade empresarial. 


\section{Introducción}

Cuando se trata de analizar los determinantes de los resultados de la actividad de las organizaciones, tiende a existir consenso sobre la importancia que en esto tienen las actividades de innovación y las de gestión del conocimiento. Así, para autores como Navas \& Nieto (2003) y Fernández, Vega \& Gutiérrez (2006), es indudable la importancia del desarrollo de estrategias de innovación y de gestión del conocimiento para mejorar la ventaja competitiva de las empresas. Sin embargo, de acuerdo con Martínez, Charterina \& Araujo (2010), los hallazgos de diversos estudios empíricos, algunos de ellos con resultados diversos y contradictorios, no permiten concluir sobre una relación empírica directa entre las actividades de innovación y las de gestión del conocimiento con la capacidad competitiva organizacional.

Por este motivo y conocedores de la importancia de la actividad innovadora y la de gestión del conocimiento para la dinámica de las organizaciones en el contexto colombiano, se realizó este estudio con el objetivo de contrastar los postulados que enfatizan la relación directa de la actividad innovadora y la de gestión del conocimiento de las empresas con variables como participación en el mercado y la rentabilidad (variables en las que se manifiesta la capacidad competitiva empresarial y que generalmente se utilizan como indicadores de ésta).

Para la realización del estudio se tomó una muestra de 241 directivos de 53 empresas grandes y medianas pertenecientes a diversos sectores de la actividad económica. La información obtenida corresponde a la actividad realizada por las empresas durante los últimos cinco años y su recolección se realizó en el periodo comprendido entre marzo y noviembre de 2010. Debido al carácter nominal de la mayoría de las variables bajo estudio, su análisis estadístico se realizó bajo las metodologías de la estadística no paramétrica.

En síntesis, los resultados del estudio indican que el $21 \%$ de las empresas manifestó haber experimentado un incremento significativo en su capacidad innovadora durante los últimos cinco años, no obstante, las pruebas de hipótesis sobre independencia de variables permiten comprobar que, con un nivel de significancia del $95 \%$, el incremento en la capacidad innovadora de las empresas no implica impacto estadísticamente significativo en los resultados finales de la dinámica empresarial tales como el crecimiento de la empresa, la participación en el mercado y la rentabilidad.

Estos resultados permiten confirmar los planteamientos de Martínez et al. (2010) donde se verifica que, para el caso de la muestra de empresas en Colombia, no existe relación directa entre la innovación de las empresas con su tasa de crecimiento, ni con la participación en el mercado o la rentabilidad, siendo estas últimas variables las dimensiones básicas en las que se manifiesta directamente la capacidad competitiva empresarial. Al respecto, estos resultados han de ser motivo de reflexión para académicos, directivos empresariales y de entidades gubernamentales al momento de analizar temas relacionados con la innovación y la gestión del conocimiento en el contexto de las organizaciones.

Este articulo esta estructurado en cuatro partes: la primera muestra el fundamento teórico que soporta el estudio, la segunda el proceso metodológico utilizado para el desarrollo del mismo, la tercera los resultados obtenidos y la cuarta las conclusiones del mismo.

\section{Marco teórico}

Para una mejor comprensión del tema, la fundamentación sobre la que se enmarca el 
estudio se ha dividido en tres subtemas así: en primera instancia, se presenta de forma breve el concepto de competitividad empresarial que es el maco central del estudio; luego, también de forma específica, se hace una presentación de los principales planteamientos sobre la relación entre gestión del conocimiento y competitividad empresarial; finalmente, se presentan los principales hallazgos sobre la relación entre innovación y competitividad empresarial.

\subsection{Aproximación al concepto de competiti- vidad empresarial}

Es importante señalar que, a pesar del significativo número de trabajos que hoy día existen sobre el tema de la competitividad, no hay consenso sobre el propio concepto. Esto se debe a la diversidad de factores que pueden intervenir en su determinación; sin embargo, hay acuerdo de que la competitividad ha de ser entendida tanto desde el enfoque micro (empresa u organización), meso (sector económico) y macro (agregado nacional) (Esser, Hillebrand, Messner \& Meyer-Stamer, 1996).

Desde la perspectiva macroeconómica, la competitividad es entendida como la capacidad de un país para, en condiciones de mercado abierto, producir bienes y servicios para los mercados nacionales e internacionales, al tiempo que se mantiene o aumenta la renta nacional (Porter, 1987). Desde la perspectiva meso, la competitividad es entendida como la capacidad de un sector para mantener o aumentar, en condiciones de libre competencia, su participación en los mercados interior y exterior, a la vez que mantiene un crecimiento satisfactorio de las rentas reales generadas por su actividad. Finalmente, desde la perspectiva microeconómica, la competitividad designa la capacidad de una empresa para producir bienes y servicios destinados a los distintos mercados donde compite, manteniendo o incrementando su cuota de participación relativa en este o estos junto con su nivel de rentabilidad (Hitt, Irelend \& Hoskisson, 2004).

$\mathrm{Al}$ respecto, es desde la perspectiva microeconómica que se realizó este estudio porque permite analizar cómo las capacidades de gestión y organización interna de cada empresa, determinan su propia competitividad (Drucker, 2000; Edvinsson \& Malone, 1997; Grant, 1996; Kogut \& Zander, 1992; Nonaka \& Takeuchi, 1998; Wiig, 2009).

\subsection{Gestión del conocimiento y competitivi- dad empresarial}

Drucker (1998) afirma que el conocimiento es el componente clave en la ventaja competitiva de cualquier empresa porque, como activo, tiene propiedades como las de multiplicarse cuando se comparte, ser la fuente de la dinámica de los demás recursos (por ejemplo, los recursos materiales no se dinamizan sino es con el uso del conocimiento) y ser un recurso con posibilidades de incremento infinitas.

Al respecto, para Quinn, Anderson \& Finkelstein (1996) la identificación de los talentos intelectuales y el aprovechamiento de su interacción dentro de la firma es lo que en última instancia determina la capacidad de competir de cualquier empresa. Sin embargo, estos autores consideran que la integración del conocimiento y su adquisición como valor, depende de las tecnologías de la información y la comunicación, al permitir sistematizarlo. En este sentido, Drucker (200o) considera que la administración de conocimiento requiere necesariamente de la tecnología de la información y la comunicación para optimizar su apropiación, generación y uso como ventaja competitiva empresarial.

De acuerdo con Grant \& Baden-Fuller (2004), la labor fundamental de la gerencia 
con el conocimiento, es articularlo en la organización para propiciar nuevas estructuras y facilitar la interacción entre las personas para explotarlo en rutinas de interacción colectivas de equipo. Según Drucker (1994), la responsabilidad del trabajador del conocimiento se centra en el desarrollo de la labor asignada, mientras que la de la gerencia es la de coordinar esa labor con la de otros especialistas.

Para autores como Nonaka \& Takeuchi (1998) y Saint-Onge (1999), el conocimiento se entiende como un activo que tiene la capacidad de generar valor y producir riqueza, por lo cual requiere ser protegido para preservar la fuente de la ventaja competitiva de la empresa. Según Bierly \& Chakrabarti (1999) y Earl (2001), las diferencias entre las firmas nacen de la forma como éstas integran, comparten y usan el conocimiento en una dinámica interactiva entre el conocimiento individual y el colectivo.

Por su parte, Boissot (1998) asegura que, mientras que la economía industrial del siglo xx giró en torno a los recursos físicos y energéticos, la competitividad de la economía de inicios de este nuevo siglo xxI se basa en el desarrollo de maneras originales y creativas de administrar los recursos y capacidades de las personas y las propias organizaciones, para construir sinergias capaces de crear valor para la organización y todos sus grupos de interés.

De acuerdo con Rivero (2002), la evidencia empírica en España muestra que, en los últimos diez años, la capacidad competitiva de las organizaciones ha estado en gran parte determinada por la capacidad de éstas para gestionar el conocimiento mediante su capacidad para identificar, adquirir, socializar o compartir, conservar, crear y usar el conocimiento como recurso estratégico. Para el mencionado autor, la evidencia muestra que para mejorar la com- petitividad de las organizaciones no basta solamente con identificar el conocimiento relevante o producirlo, sino que es preciso saberlo utilizar de la forma más eficaz, es decir, las organizaciones requieren saber gestionar el conocimiento y la pertinencia del mismo, más que producirlo. Al respecto, para McAdam \& Reid (2001) las empresas que saben gestionar el conocimiento (identifican, crean, incorporan, difunden y usan adecuadamente este recurso), según el contexto en el que actúan, tienden a ser más competitivas que aquellas que no lo saben.

Según un estudio de la KPMG (2007), el $81 \%$ de las grandes y medianas empresas en Estados Unidos, Europa y Asia, afirman tener o por lo menos están considerando adoptar un sistema administrativo basado en el conocimiento.

En este mismo sentido, el estudio realizado por Andreu, Baiget \& Salvaj (2004) sobre gestión del conocimiento como estrategia competitiva en las empresas españolas, muestra que el $67 \%$ de estas actualmente realizan actividades de gestión del conocimiento y que otro $38 \%$ aseguran tener previsto poner en marcha proyectos de este tipo en un breve tiempo, especialmente en lo relacionado con soluciones tecnológicas. Igualmente, este estudio señala que el $92 \%$ de las empresas consideran a la gestión del conocimiento como la base de su competitividad porque proporciona ventajas diferenciadoras duraderas. Finalmente, el estudio indica que el $78 \%$ de los empresarios o directivos consideran que la buena gestión del conocimiento impacta de forma directa en la mejora de la calidad de los bienes o servicios, el $75 \%$ en la satisfacción de los clientes y el $70 \%$ en la capacidad de respuesta efectiva a los cambios del entorno.

Igualmente para el caso colombiano, estudios recientes sobre gestión del conocimiento indican que los empresarios y 
directivos de medianas y grandes empresas, tienden a estar de acuerdo en que el conocimiento es la principal fuente de competitividad empresarial en el nuevo orden económico mundial en el siglo xxI (Bernal, 2010; Bernal, Turriago \& Sierra, 2010; Blanco \& Bernal, 2009).

\subsection{Innovación y competitividad empresarial} Para Porter (1987) la innovación es el elemento clave que explica la competitividad de las naciones, los sectores empresariales y las propias empresas, al afirmar que la competitividad de una nación depende de la capacidad de su industria para innovar y que una empresa logra ventaja competitiva cuando realiza de forma sostenible innovaciones para el mercado.

Por su parte, Corona (2008) sostiene que la competitividad es una variable multifactorial que tiene relación con la formación empresarial, las habilidades administrativas, laborales y productivas, la gestión, la innovación y el desarrollo tecnológico. Según este autor, para explicar la competitividad empresarial se requiere de una base multifactorial y sistémica de la propia dinámica empresarial. Así, la competitividad requiere arreglos institucionales diversos que incluyen las estrategias de mercado, la dinámica de la competencia y las estrategias respecto a las capacidades y recursos internos a la empresa.

En este sentido, la innovación es una condición necesaria, pero no suficiente para alcanzar la competitividad, porque una empresa no compite contra otra u otras empresas sino contra toda la base institucional, de apoyo financiero, de generación y aplicación de tecnología, de subsidios y apoyos que generan las naciones; es decir, que las empresas expresan la competitividad de los sistemas productivos en los que están inmersas.
De acuerdo con Bueno (2002), la competitividad desde las concepciones macro y meso explican la competitividad entre las naciones y los sectores industriales pero no la heterogeneidad de los resultados entre empresas del mismo sector. Esta heterogeneidad es explicada por una ventaja basada en el dominio por parte de una empresa de una característica, habilidad, recurso o conocimiento diferencial que incrementa su eficiencia y le permite distanciarse de la competencia.

Así, para Clark (1987) y Dosi, Teece \& Chitry (1998), las virtudes y ventajas de la innovación en una empresa dependen de sus características y estrategias. Agregan estos autores que, si bien existe evidencia de que la innovación es vital para el crecimiento y para el mantenimiento de la competitividad, no lo es tanto el qué, por qué y cuándo debe hacerse para que sea éxito o fracaso, ya que la base de la posición competitiva de una empresa radica en sus peculiaridades y capacidades para influir en los recursos, habilidades y conocimientos existentes.

De otra parte, según Pascale (2005) y Larios (2002), estudios sobre las relaciones existentes entre el esfuerzo innovador y el éxito del negocio, muestran que las compañías líderes no son solamente mejores en cuanto a beneficios, tasa de retorno, facturación por empleado y capacidad para adoptar más rápidamente las nuevas tecnologías, sino que están más orientadas al mercado, dedican mayores esfuerzos al desarrollo de su capital humano, tienen mejores e importantes relaciones con sus clientes, una gestión más eficaz y altas competencias en marketing.

De acuerdo con INNO-Alemania (2009), en uno de sus estudios sobre las relaciones entre la empresa y organismos externos, en particular, con clientes, proveedores, universidades, organismos de investigación y 
consultoras; las empresas que poseen fuertes vínculos con esas entidades externas tienen claramente mayor éxito y son más competitivas que empresas que son innovadoras pero con pocos vínculos con su entorno externo.

De hecho, la OCDE (2008) identifica que, en el nuevo entorno competitivo, el comportamiento innovador de las empresas está determinado por un amplio espectro de conocimientos e informaciones sobre temas asociados al entorno, como las preferencias de los consumidores, sistemas de comunicación, relaciones de producción, mercados, sistemas de distribución, publicidad en diferentes ambientes culturales, etc.

Según el enfoque de recursos y capacidades (Teece, Pisano \& Shuen, 1997), un elemento clave de la capacidad de innovación de las empresas reside en su habilidad para desarrollar y reconfigurar las competencias internas con el fin de responder a los cambios del entorno. El estudio de la relación entre flexibilidad de recursos humanos e innovación es importante porque la innovación en las empresas depende cada vez más de la integración de conocimientos internos y externos. Para que las empresas tengan éxito en su proceso de innovación, no solo han de saber explotar bien sus recursos internos, sino que también han de ser capaces de explorar y asimilar conocimientos que no se hayan desarrollado internamente (Rothaermel \& Hess, 2007).

Por lo antes mencionado, este estudio buscó indagar sobre el papel de las actividades de innovación y las de gestión del conocimiento en la dinámica competitiva de las empresas localizadas en la ciudad de Bogotá, con el propósito contribuir con la sistematización de información en este campo en el medio colombiano y con la intención de proveer de insumos a directivos, empresarios, académicos y gobernantes para la formulación de estrategias que puedan contribuir a mejorar la capacidad competitiva empresarial del país.

\section{Metodología}

Este estudio es de carácter analítico y fue realizado con una muestra de empresas grandes y medianas pertenecientes a diversos sectores de actividad económica localizadas en la ciudad de Bogotá y municipios aledaños. Para la selección de las empresas se tuvieron en cuenta tres criterios: el primero, y el más importante, fue el que estas mostraran haber realizado durante los últimos cinco años actividades de innovación y de gestión del conocimiento (para ello se utilizaron los resultados de estudios previos ${ }^{3}$ relacionados con temas de innovación y gestión del conocimiento en las organizaciones en el contexto de aplicación del estudio); el segundo fue que se tratase de empresas medianas o grandes en tamaño, esto porque es en este tamaño de empresas donde se han identificado actividades simultáneas de innovación y gestión del conocimiento; finalmente, el tercer criterio fue que las empresas deberían tener al menos diez años de actividad continua.

Con el cumplimiento de esos criterios se identificaron 87 empresas, las cuales fueron contactadas para ser informadas sobre los objetivos del estudio. Finalmente, se logró aplicar las encuestas en 53 de ellas, a un total de 241 directivos (la idea de entrevistar varios directivos por empresa fue la de reducir sesgos de percepción por parte de estos en relación con las variables objeto de análisis).

El instrumento utilizado para la obtención de la información fue un cuestionario de encuesta que buscaba identificar las actividades de innovación (de productos, de procesos, de marketing y organizacionales)

3 Hace referencia a los estudios de Bernal et al. (2010) y Blanco \& Bernal (2009). 
y las de gestión del conocimiento (identificación, registro, incorporación, creación y uso de conocimiento como estrategia organizacional) realizadas por la empresas durante los últimos cinco años de actividad y los impactos que esas actividades generaron en la rentabilidad y/o la participación del mercado de las empresas.

Antes de la aplicación definitiva, el formato de la encuesta fue previamente validado mediante una muestra piloto con trabajo de campo en cinco empresas, y también con el método de concepto de expertos. Las encuestas fueron aplicadas durante el periodo marzo-noviembre de 2010. ${ }^{4}$

El análisis estadístico de la información se realizó mediante el cálculo de frecuencias y el inferencial con prueba de hipótesis para independencia de variables aplicando el Chi-cuadrado de Pearson con factor de corrección por continuidad, con un nivel de confianza del $95 \%$.

\section{Resultados}

Respondiendo al objetivo del estudio, a continuación se presentan los resultados de la encuesta aplicada a los 241 directivos de las 53 empresas que conformaron la muestra. Primero se analiza la relación entre las actividades de gestión del conocimiento y la competitividad de las empresas y luego la relación entre las actividades de innovación con dicha competitividad.

\subsection{Relación entre gestión del conocimiento $y$ competitividad empresarial}

En general, los resultados indican que del total de las empresas participantes en el estudio, el 20\% manifestó haber experimentado, en los últimos cinco años, un incremento significativo en su capacidad competitividad (capacidad para mantener

4 La encuesta puede ser accedida bajo solicitud directa a los autores. o ganar participación en el mercado manteniendo o aumentando la rentabilidad); el $25 \%$ indicó haber logrado un incremento medio, el $10 \%$ un incremento bajo y el $45 \%$ ningún incremento.

En cuanto a las actividades de gestión del conocimiento, para el conjunto de las empresas las fuentes más importantes de obtención y uso de este recurso para el desarrollo de sus actividades son los estudios de mercado (68\%), los contactos permanentes con los clientes $(47 \%)$, los contactos con los proveedores ( $41 \%)$, la asistencia a ferias y exposiciones (43\%) y las inteligencias de mercado (39\%). Por su parte, las herramientas más usadas para esa gestión del conocimiento son los sistemas integrales de información (81\%) y las redes de práctica de conocimiento $(63 \%)$ y, las menos usadas son los mapas de conocimiento (22\%) y las ontologías de conocimiento ( $7 \%$ ).

Igualmente, para las empresas que manifestaron haber logrado incrementos significativos en su capacidad competitiva, las herramientas de gestión del conocimiento más empleadas son la gestión documental y de contenidos ( $72 \%)$, seguidas por los sistemas integrales de información (57\%), en tanto que la menos empleada es la ontología de conocimiento (11\%). Los impactos del uso de esas herramientas para estas empresas se refleja principalmente en la mejora de: la calidad de los productos y/o servicios (82\%), los procesos que se realizan en la empresa (78\%), las relaciones con los clientes (71\%), los niveles de control y autonomía laboral (53\%) y el clima laboral (47\%).

Ahora, al analizar los resultados de las empresas en las que sus directivos manifestaron mejoras en la capacidad competitiva, de acuerdo con el estadístico Chi-cuadrado con nivel de significación del $95 \%$, se encontró que no existe interdependencia entre las actividades de gestión del conocimiento 
realizadas por esas empresas en los últimos cinco años y la mejora de su capacidad competitiva (ver Tabla 1).

\subsection{Relación entre actividades de innovación y capacidad competitiva}

En lo referente a la innovación, los datos del estudio para el conjunto de la muestra de empresas, indican que las principales actividades relacionadas con la innovación realizadas en los últimos cinco años de actividad fueron, en su orden de importancia, la mejora en los proceso productivos (64\%), cambios en las actividades de marketing (46\%), la diversificación en la gama de bienes/servicios ofrecidos (43\%) y la mejora o cambios en los procesos administrativos (37\%).

También, los datos indican que el impacto de las actividades de innovación realizadas por las empresas se suelen reflejar en la gama de productos y/o servicios (62\%), la reducción de consumo de materias primas $(57 \%)$, la adaptación a los estándares internacionales (53\%), la mejora en la capacidad productiva $(44 \%)$, la reducción de impacto del medio ambiente (31\%), la reducción de costos (27\%), el acceso a nuevos mercados $(27 \%)$ y la rentabilidad de la empresa (18\%).

De otra parte, cuando se analiza la relación directa entre las actividades de innova- ción realizadas por las empresas durante los últimos cinco años y la mejora de su capacidad competitiva (medida en términos de participación del mercado con rentabilidad), se encontró que, en general, solo siete (13\%) de las 53 empresas evidencian dicha relación. $\mathrm{Al}$ respecto, cuando se indaga a los directivos sobre los factores determinantes de la competitividad de sus empresas, los resultados de este estudio indican que esos factores son las preferencias de los clientes por los bienes o servicios de las empresas (75\%), el adecuado uso que hacen las empresas de sus recursos y capacidades (65\%) y las condiciones externas del mercado (45\%); mientras que los factores menos relevantes son los apoyos del gobierno $(25 \%)$ y las relaciones del país con mercados internacionales $(25 \%)$ (ver Gráfico 1).

\section{Conclusiones}

Aunque indudablemente en la generalidad de la bibliografía sobre los campos de la gestión del conocimiento y la innovación se suele afirmar la relación directa entre estas variables y la capacidad competitiva empresarial, los resultados de este estudio muestran que, para el caso de la muestra analizada en las empresas localizadas en la ciudad de Bogotá, dicha relación directa es muy baja, casi inexistente. Lo anterior

Tabla 1. Pruebas Chi-cuadrado* para la relación entre actividades de gestión del conocimiento y los principales indicadores de competitividad de las empresas

\begin{tabular}{|c|c|c|c|c|}
\hline $\begin{array}{l}\text { Resultados de las } \\
\text { empresas }\left(X_{\mathrm{j}}\right)\end{array}$ & Rentabilidad & $\begin{array}{l}\text { Participación } \\
\text { en el mercado }\end{array}$ & $\begin{array}{c}\text { Incursión en } \\
\text { nuevos mercados }\end{array}$ & $\begin{array}{l}\text { Capacidad } \\
\text { innovadora }\end{array}$ \\
\hline $\begin{array}{l}\text { Gestión documental } \\
\text { y de contenidos }\end{array}$ & 0,55 & 0,10 & 0,134 & 0,56 \\
\hline $\begin{array}{l}\text { Sistemas integrales } \\
\text { de información }\end{array}$ & 2,67 & 0,43 & 0,167 & 2,1 \\
\hline
\end{tabular}

Nota. ${ }^{\star}$ Con un nivel de confianza del 95\% la hipótesis nula se acepta para valores $0<X^{2}<3,84$.

Fuente: Elaboración propia. 
Análisis de la relación entre la innovación y la gestión del conocimiento con la competitividad empresarial en una muestra de empresas en la ciudad de Bogotá

Gráfico 1. Factores considerados por las empresas como de alto impacto en su competitividad

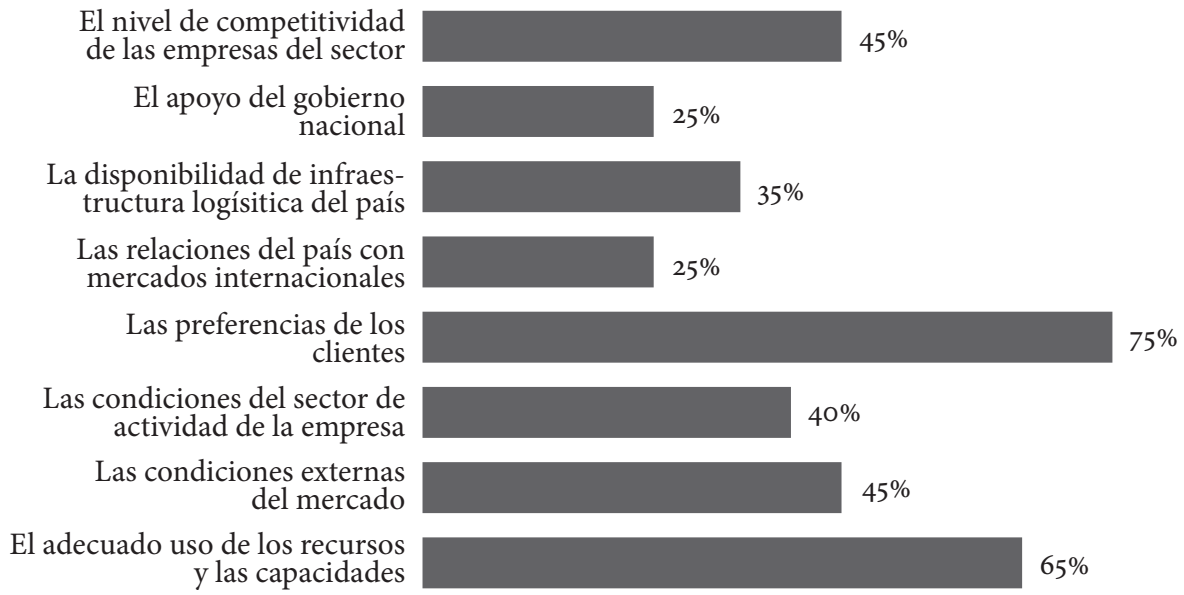

Fuente: Elaboración propia.

corrobora los resultado de estudios previos realizados en España (Martínez et al., 2010). Sin embargo, existe un alto impacto de esas variables en la mejora de los procesos de producción de los bienes o servicios, en las relaciones con los clientes y en la reducción del consumo de materias primas.

Lo anterior sugiere que, para el caso de empresas en contextos como los de Colombia (economía emergente), las actividades de innovación y las de gestión del conocimiento, más que reflejarse en resultados finales de la actividad empresarial (participación en el mercado y las utilidades), se reflejan en procesos intermedios de su dinámica, por lo menos en el corto plazo.

De otra parte, los resultados del estudio también estimulan la reflexión a favor del enfoque multifactorial de la capacidad competitiva de las empresas (Bueno, 2002; Corona, 2008; Larios, 2002; OCDE, 2008) que afirma que esta capacidad competitiva está en función de la interrelación entre diversos factores, donde las actividades de innovación y las de gestión del conocimiento, son unos de ellos y no los únicos. Esto es posible porque factores como la calidad de los bienes o servicios ofrecidos, las competencias administrativas y técnicas de las personas que laboran en la empresa, la disponibilidad y el uso de las tecnologías de la información y las comunicaciones (TICs), entre otros, también son altamente determinantes de esa capacidad competitiva.

Sin embargo, también es claro que el aprovechamiento estratégico de estas variables por parte de todos los colaboradores de la empresa es fundamental para crear ventajas competitivas sostenibles para todo tipo de organizaciones (independientemente de su tamaño, sector, constitución jurídica, etc.). Estas ventajas deben ser aplicables en cualquier contexto, dado que son precisamente la innovación y la gestión del conocimiento, las variables o factores capaces de proveer rasgos distintivos y generadores de valor compartido sostenible en cualquier organización; especialmente si se considera el actual ambiente de los negocios, caracterizado por una cada vez mayor globalización 
de mercados, competitividad, incertidumbre y cambio (Bueno, 2002; Drucker, 2000; Nonaka \& Takeuchi, 1998; Wiig, 2009).

En este sentido, los resultados de este estudio son un factor para la reflexión en el ámbito académico, empresarial y gubernamental que invita a repensar la concepción tradicional de la relación causal directa entre las actividades de innovación o de gestión del conocimiento con la capacidad competitiva empresarial (particularmente en los resultados finales), independientemente de la presencia o no de otra variables pues esta concepción no ha sido avalada por la evidencia empírica.

Finalmente, es importante señalar que, dada la limitada amplitud de la muestra tanto por el número de empresas participantes del proyecto como por el contexto geográfico en donde éste se realizó, es recomendable desarrollar nuevos estudios con tamaño de muestra más significativo por número de empresas y contexto geográfico, lo que permitirá disponer de resultados más concluyentes sobre el objetivo del estudio. Igualmente, es importante que en esos estudios se tenga en cuenta criterios como la existencia en las empresas participantes de indicadores de impacto de las actividades de innovación y gestión del conocimiento en el corto, mediano y largo plazo.

\section{Referencias bibliográficas}

Andreu, R., Baiget, J. \& Salvaj, E. (2004). Gestión del conocimiento y competitividad en la empresa española. Barcelona: CapGemini-IEsE.

Bernal, C. (2010). Modelo integral de administración basada en el conocimiento. Tesis no publicada para obtener el titulo de Doctor en Administración de Negocios,
Newport University, Newport News, va, Estados Unidos.

Bernal, C., Turriago, A. \& Sierra, H. (2010). Aproximación a la medición de la gestión del conocimiento empresarial. Revista Administer, 16, 31- 49.

Bierly, P. \& Chakrabarti, A. (1999). Generic Knowledge Strategies in the U.S. Pharmaceutical Industry. Strategic ManagementJournal, 17(1), 123-135.

Blanco, C. \& Bernal, C. (2009). Percepciones sobre la gestión del conocimiento por parte de una muestra de directivos. Cultura, Tecnología y Patrimonio, 4(7), 81-97.

Boissot, M.H. (1998). Knowledge asset. Oxford, uk: Oxford University Press.

Bueno, E. (2002). Dirección estratégica basada en conocimiento: Teoría y práctica de la nueva perspectiva. En P. Morcillo, \& J. Fernández-Aguado (coord.), Nuevas claves para la dirección estratégica (pp. 91 166). Madrid: Ariel.

Clark, K.B. (1987). Investment in new technology and competitive advantage. En D.J. Teece (Ed.), The competitive challenge (pp. 59-82). Cambridge, MA: Ballinger.

Corona, L. (2008). Innovación y competitividad empresarial. Revista de la Facultad de Economía-BUAP, 20.

Dosi, G., Teece, D. \& Chitry, J. (1998). Technology, Organization and Competitiveness. Londres: OxfordUniversity Press.

Drucker, P. (1994). Gerencia para el futuro. Bogotá: Norma.

Drucker, P. (1998). La sociedad postcapitalista. Bogotá: Norma.

Drucker, P. (2000). Retos de la gerencia para el siglo XXI. Bogotá: Norma.

Earl, M. (2001). Knowledge Management Strategies: Toward Taxonomy. Journal of Management Information Systems, 18(1), 215-233.

Edvinsson, I. \& Malone, M. (1997). El capital intelectual. Barcelona: Gestión 2000. 
Esser, K., Hillebrand, W., Messner, D. \& Meyer-Stamer, J. (1996). Competitividad sistémica: nuevo desafío para las empresas y la política. Revista CEPAL, 59, 39-52. Fernández, I., Vega, J. \& Gutiérrez, A. (2006). Estrategias de innovación de las empresas manufactureras españolas. Valencia: Instituto de Gestión de la Innovación y del Conocimiento. Recuperado el 4 de agosto de 2010, de http://digital.csic.es/ bitstream/10261/10028/1/Articulo\%20 economistas.pdf

Grant, R.M. (1996). Dirección Estratégica. Conceptos, técnicas y aplicaciones. Madrid: Civitas.

Grant, R.M. \& Baden-Fuller, C. (2004). A Knowledge accessing theory of strategic alliances. Journal Management Studies, 41(1), 61-79.

Hitt, M., Ireland, D. \& Hoskisson, R. (2004). Administración estratégica: Competitividad y conceptos de globalización. México: Thomson.

INNO-Alemania. (2009). Innovación y Competitividad: la estrategia alemana. Recuperado el 9 de febrero de 2010 , de http://www.ahk-colombia.com/ fileadmin/ahk_kolumbien/Dokumente/ DB59_-_internet.pdf

Kogut, B. \& Zander, U. (1992). Knowledge of the Firm, Combinative Capabilities and Replication of Technology. Organization Science, 3(3), 383-397.

KPMG Consulting. (2007). Knowledge Management Research Report. Londres: KPGM International.

Larios, F. (2002). Innovación, ¿Factor de competitividad? Revista: MadrI+d. Recuperado el 27 de julio de 2009, de http:// www.madrimasd.org/informacionidi/ revistas/Numero2/aula.asp

Martínez, R., Charterina, J. \& Araujo, A. (2010). Un modelo causal de competitividad empresarial planteado desde la visión de los recursos y capacidades: Capacidades directivas de innovación, marketing y calidad. Investigaciones Europeas, 16(2), 165-188. Recuperado el 21 de junio de 2010, de http://www.aedem-virtual.com/ articulos/iedee/v16/162165.pdf

McAdam, R. \& Reid, R. (2001). SME and Large organization of Knowledge management: comparisons and Contrasts. Journal of Knowledge Management, 5(3), 231-247.

Navas, J. \& Nieto, M. (2003). Estrategias de innovación y creación de conocimiento tecnológico en las empresas industriales españolas. Madrid: Civitas.

Nonaka, I. \& Takeuchi, H. (1998). The knowledge creating company: how Japanese companies create the dynamics of innovation. Oxford, uK: Oxford University Press.

OCDE. (2008). Ciencia, Tecnología e Industria. Recuperado el 13 de noviembre de 2009, de http://www.oecd.org/sti/innovationinsciencetechnologyandindustry/41553412.pdf

Pascale, R. (2005). Gestión del conocimiento, innovación y productividad. Exploración del caso de la iwndustria manufacturera uruguaya. Recuperado el 7 de enero de 2010, de http://www.uoc.edu/in3/dt/esp/ pascale0605.html

Porter, M. (1987). Ventaja competitiva de las naciones. Buenos Aires: Vergara.

Quinn, J.B., Anderson, P. \& Finkelstein, S. (1996). La Gestión del Intelecto Profesional: Sacar el Máximo de los Mejores. Harvard Deusto Business Review, 75, 4-17. Rivero, S. (2002). Claves y pautas para comprender e implantar la gestión del conocimiento. Madrid: Fundación Escuela de Ingenieros de Bilbao - Socintec.

Rothaermel, F. \& Hess, A. (2007). Building dynamic capabilities: Innovation driven by individual, firm, and network-level effects, Organization Science, 18(6), 898- 
921. Recuperado el 12 de septiembre de 2010 , de http://ubr.universia.net/ pdfs_web/UBR_2400930.pdf.

Saint-Onge, H. (1999 [1996]). Tacit knowledge: The key to the strategic alignment of intellectual capital. Strategy \& Leadership, 24(2), 10-14.

Teece, D.J., Pisano, G. \& Shuen, A. (1997). Dynamic capabilities and strategic management. Strategic Management Journal, 18(7), 509-533.

Wiig, K. (2009). Knowledge Management for the Competitive Enterprise. Arlington, TX: Knowledge Research Institute. 